\title{
Study of Porosity Gradient in Released Porous Silicon Microstructures
}

\author{
$\underline{\text { Yaman Afandi }}^{1,2, *}$, Giacinta Parish ${ }^{1}$ and Adrian Keating ${ }^{2}$ \\ ${ }^{1}$ School of Electrical, Electronic and Computer Engineering, University of Western Australia, 35 Stirling Hwy, Crawley, \\ Western Australia 6009, Australia. \\ ${ }^{2}$ School of Mechanical and Chemical Engineering, University of Western Australia, 35 Stirling Hwy, Crawley, Western \\ Australia 6009, Australia. \\ *yaman.afandi@research.uwa.edu.au
}

Porosity gradients are a key factor limiting the application of porous silicon (PS) in micromachined structures. In this work, the in-depth porosity profile of PS was studied by measuring the average porosity for samples grown at both constant current density and varied current density; the latter designed to compensate vertical porosity gradient effects. For constant current density, the results showed that the porosity increases from the top surface toward the Si/PS interface. This result is applicable to samples less than 4- $\mu$ m thick. The varied current density investigations with current density reduced over the duration of anodisation indicated that two recipes at $\Delta \mathrm{I}=10$ $\& 12 \mathrm{~mA} / \mathrm{cm}^{2}$ resulted in a relatively low porosity gradient and low tensile residual stress. Using these two recipes, released MEMS-based PS microstructures were fabricated. The results showed that the recipe using a current density variation of $\Delta \mathrm{I}=10 \mathrm{~mA} / \mathrm{cm}^{2}$ (where $\mathrm{I}_{\text {initial }}=20 \mathrm{~mA} / \mathrm{cm}^{2}$ and $\mathrm{I}_{\text {final }}=10 \mathrm{~mA} / \mathrm{cm}^{2}$ ) was the best approach to produce microstructures with a peak to valley (PV) flatness of 200 nm. This study provides a pathway to create released, MEMS-based resonant cavity optical devices using only a single material (Si) platform.

\section{Introduction}

Porous silicon (PS) provides an important platform for MEMS-based sensing applications due to its morphological and physical properties [1]. The simplest method to form PS thin films is by electrochemical dissolution of silicon in hydrofluoric acid-based solutions. However, this technique leads to a non-uniformity in the porosity in the direction of film growth which causes a deformation in MEMS-based PS devices, affecting optical and mechanical device performance [2]. It is well known, such as that by Valalaki et al. [3], that increasing the anodisation current density leads to an increase in the porosity. By knowing the in-depth porosity profile, the porosity can be compensated by varying the current during anodisation. Lascaud et al. [4] found that $80-\mu \mathrm{m}$ thick multilayered PS with decreasing porosity from $55 \%$ to $35 \%$ can be achieved by adjusting the current density during the electrochemical etching. However, adjusting the current density during anodisation to achieve uniform porosity, and accordingly, flat MEMSbased PS structures has received little attention. The exception is a recent study by Xiao et al. that showed that using an exponential current variation during anodisation produced improved flatness in PS microstructures [2].

The aim of this work is to study the porosity profile with depth to design a recipe for the current variation during anodisation that best compensates the porosity gradient and hence produce films with low stress gradient. Ultimately, the goal is to be able to create more complex microstructures such as released (tunable) PS resonant cavities.

\section{Experimental}

P-type silicon wafers (100) with resistivity of 0.08-0.12 $\Omega . \mathrm{cm}$ were used to create our porous films. Our work focuses on PS fabricated from moderately doped silicon to allow low-loss optical sensors to be created in the infrared.

PS thin films were formed in 15\% HF/ethanol solution at room temperature. Initially, different thicknesses of PS thin films were fabricated at a constant current density of I $=10 \mathrm{~mA} / \mathrm{cm}^{2}$ to study the in-depth porosity profile.

Subsequently, a range of current variations $(\mathrm{I}(\mathrm{t}))$ were used to fabricate PS thin films with a target thickness of 2.5 $\mu \mathrm{m}$. Initially, the porosity is assumed to linearly increase with depth for films less than $3 \mu \mathrm{m}$ thick. The desired current density was deduced from the estimated average porosity. Various current density profiles were designed and trialled. The change in applied current during anodisation was used to explore the induced gradient and to assess the ability to compensate the inherent gradient $d P_{o} / d h$. Different PS films were produced with different film porosity compensating gradients $(d P / d h)$. Fig. 1 shows one profile of current density to fabricate a film of $2.5-\mu \mathrm{m}$ thick using current density starting at $20 \mathrm{~mA} / \mathrm{cm}^{2}$ and ending at $10 \mathrm{~mA} / \mathrm{cm}^{2}$.

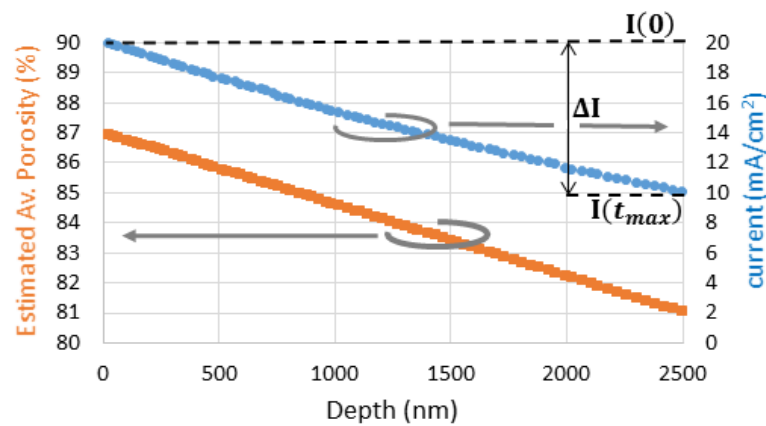

Figure 1: The left axis shows the estimated average porosity along the depth for a $2500-\mathrm{nm}$ thick film. The right axis gives the varied current density used to compensate the inherent porosity gradient.

After formation, the PS films were annealed in a $\mathrm{N}_{2}$ atmosphere at $600{ }^{\circ} \mathrm{C}$ at $1000 \mathrm{sccm}$ to create a passivation layer that protects PS from oxidation and susceptibility to alkaline solutions [4]. A comparison between annealing times of 6 minutes and 48 minutes was undertaken. A genetic algorithm was used to fit the reflectance spectrum to a Bruggeman model [5] to extract calculated porosity 
gradient and refractive index $(n)$. The residual stress $(\sigma)$ measurement was obtained from film bowing measurements using Stoney's equation [6]. For two of the porosity gradient compensated structures that were produced, released PS microstructures were fabricated using a process that has been reported previously [2].

Scanning electron microscopy (SEM) using a Verios XHR SEM and a Zeiss SEM was used to characterise the porosity at the top surface of PS thin films and at the back side of the released PS microstructures. Surface profilometry measurements were performed on released beams using an optical profilometer from Zygo Inc.

\section{Results}

Fig. 2 demonstrates measured average porosity of asfabricated PS thin films of different thickness formed using a constant current of $10 \mathrm{~mA} / \mathrm{cm}^{2}$. The obtained curve allows a good assessment of the in-depth porosity profile in the thickness range of interest.

Fig.2a indicates that the average porosity decreases for films up to 2000-nm thick. Correspondingly, Fig.2b shows that the porosity gradient is high for the film $100-\mathrm{nm}$, suggesting that the high average porosity arises from a high porosity at the interface. The porosity gradient was extracted from the best fit to the optical reflectance spectrum over a wavelength range of $500 \mathrm{~nm}-900 \mathrm{~nm}$, using a model that defines the gradient as:

$$
\frac{\mathrm{dP}}{\mathrm{dh}}=\frac{\mathrm{P}_{\text {Si/PS interface }}-\mathrm{P}_{\text {Top surface }}}{\text { Film thickness }}
$$

The SEM images, in Fig.3, show that the size of pores becomes wider and the pore distribution becomes more inhomogeneous on the top of the film as the total sample thickness increases. Long exposing to HF electrolyte during the fabrication of thicker films is attributed to the increased pore size (and porosity) at the top surface. At a thickness above $4000 \mathrm{~nm}$, the average porosity increases suggesting that the actually (localized) porosity at the interface is considerably higher. The high porosity at the interface is attributed to the depletion of the HF during the anodisation [7]. At a thickness of 5500-nm, the sample starts detaching from the substrate. Given the increased porosity occurs over a relatively short region of $4000-5500 \mathrm{~nm}$, we believe considerable depletion of the HF is occurring in this region, such that current adjustment cannot appropriately compensate for the increased porosity. A high porosity at the top surface due to chemical etching and high porosity at the interface due to HF depletion effectively leads to a zero porosity gradient, as measured in Fig.2b.

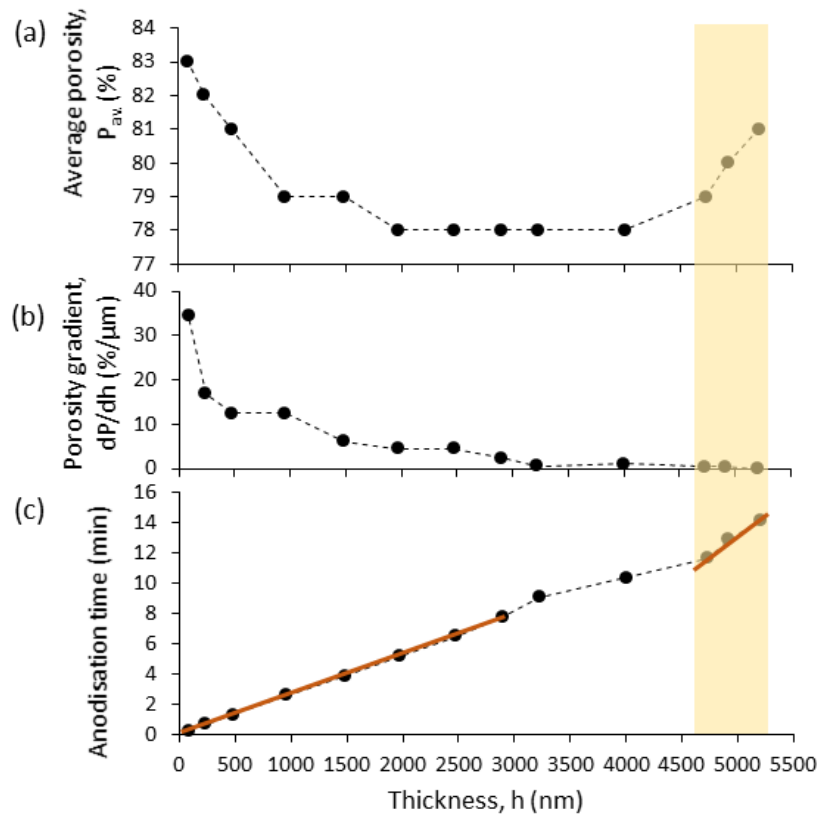

Figure 2: (a) The measured average porosity, (b) porosity gradient and (c) anodisation time at different thicknesses. Films fabricated with constant current of $10 \mathrm{~mA} / \mathrm{cm}^{2}$.

Fig.2c shows our measurements of the time to achieve a specific optical thickness from our samples. In the range from 0-3000 nm, the etch rate is extremely uniform. Given that the porosity formed during anodization and etch rate are highly correlated, it is unlikely the higher top surface porosity observed in Fig. 3 is a result of the anodization current or HF concentration changes. The constant etch rate (slope) in Fig.2c up to $300 \mathrm{~nm}$ supports the argument that chemical etching is increasing the top film porosity, which is in line with previous studies showing pore widening at the top surface leading to increase porosity in this region [8]. However, the etch rate does decreases for film thicknesses above $4500 \mathrm{~nm}$, suggesting a high porosity in this region.

The very uniform porosity between 2000-4500 nm shown in Fig.2a suggests the two processes (chemical dissolution and HF depletion) are largely balanced in this region. However, our final process step for the release of MEMS devices involves electrochemical etching of the PS/Si interface (see Fig.4 and Fig.5 later) [2]. The initiation of this process may also increase the porosity of the backsurface, making the porosity gradient (and hence stress gradient) in the film worse. 


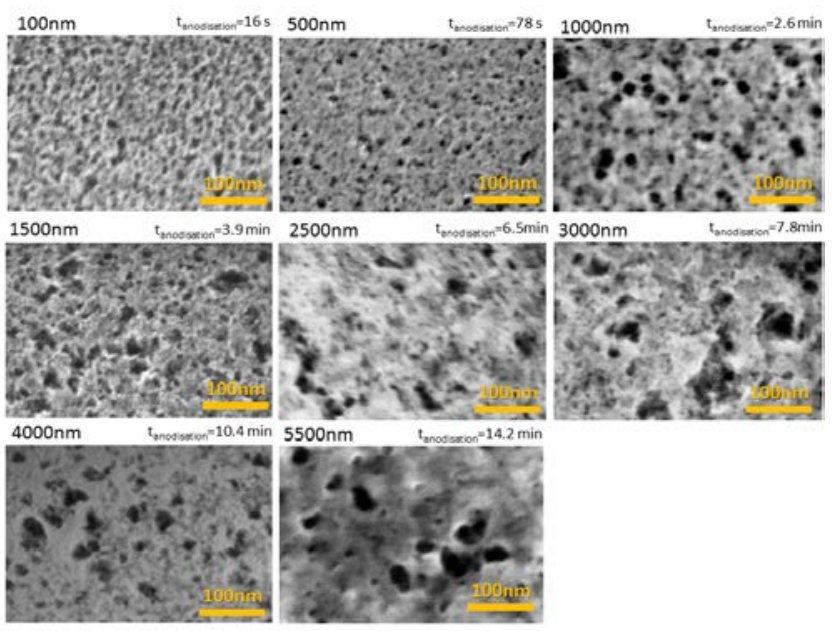

Figure 3: SEM image of the top surface of PS at different thicknesses using constant current $I=10 \mathrm{~mA} / \mathrm{cm}^{2}$.

In-depth porosity was compensated by applying varied current (I(t)), starting at $20 \mathrm{~mA} / \mathrm{cm}^{2}$ and ending at a lower current density. Decreasing the current leads to reduced porosity as indicated in Fig.1, which reduces the impact of the large increase in porosity that occurs at the PS/Si interface due to a decrease in HF concentration during the anodisation. To find the best recipe of current density that can produce flat microstructures, the porosity gradient along with the residual stress were measured at different varied current densities. For simplicity, each current recipe was represented in Fig.4g by Eq.2:

$$
\Delta \mathrm{I}=\mathrm{I}\left(\mathrm{t}_{0}\right)-\mathrm{I}\left(\mathrm{t}_{\max }\right)
$$

where $\mathrm{I}\left(\mathrm{t}_{0}\right)$ is the starting current density of $20 \mathrm{~mA} / \mathrm{cm}^{2}$, and $I\left(t_{\max }\right)$ is the final current density. All the films have the same thickness of $2500 \mathrm{~nm}$.

Using constant anodisation current density of $\mathrm{I}=20$ $\mathrm{mA} / \mathrm{cm}^{2}, \Delta \mathrm{I}=0$, caused the sample to be detached at a film depth of $2500 \mathrm{~nm}$, indicating very high porosity at Si/PS interface. Fig.4 (a \& b) show SEM images of both top side and back side of the detached sample, which clearly show that the porosity at the back side is higher than the top side. Whether this is due to HF depletion during anodisation or residual anodisation on the back-surface occurring just prior to electropolishing requires further investigation.

Varied current densities starting at $20 \mathrm{~mA} / \mathrm{cm}^{2}$ and ending at 18,16, 14 and $12 \mathrm{~mA} / \mathrm{cm}^{2}$ were applied and presented in Fig.4g using $\Delta \mathrm{I}=2,4,6$ and $8 \mathrm{~mA} / \mathrm{cm}^{2}$ respectively. These samples produced partly detached samples with rough surfaces, which resulted in poor reflectance spectra, and consequently, difficulty in extracting the porosity data. The stress at these values also has a high uncertainty.

Only two samples using varied current densities at $\Delta \mathrm{I}=$ $10 \& 12 \mathrm{~mA} / \mathrm{cm}^{2}$ (starting at $20 \mathrm{~mA} / \mathrm{cm}^{2}$ and ending at 6 or $4 \mathrm{~mA} / \mathrm{cm}^{2}$ respectively) produced results with low porosity and low stress as shown in Fig.4(g \& h). Using these two recipes, the porosity gradient and the residual stress were measured again after annealing the sample for 6 minutes and for 48 minutes, as shown in Fig.4h. The measured data suggests that annealing the sample for 6 minutes is preferable as the stress is slightly tensile, as shown in
Fig.4h, while annealing the sample for 48 minutes produced compressive stress that causes bowing to microstructures.

SEM images of the pores distribution on the back side and the top side of released structures using $\Delta \mathrm{I}=10$ and 12 $\mathrm{mA} / \mathrm{cm}^{2}$, shown in Fig.4(c \& d) and Fig.4(e \& f), respectively, give an estimation of how the porosity was compensated with depth. While the pores size is almost the same at the top surfaces of both $\Delta \mathrm{I}=10 \& 12 \mathrm{~mA} / \mathrm{cm}^{2}$ samples, the pores size at the back side of $\Delta \mathrm{I}=10 \mathrm{~mA} / \mathrm{cm}^{2}$ sample are slightly larger than on the backside of $\Delta \mathrm{I}=12$ $\mathrm{mA} / \mathrm{cm}^{2}$ sample, suggesting that better porosity compensation at the back-surface occurred for the $\Delta \mathrm{I}=10$ $\mathrm{mA} / \mathrm{cm}^{2}$ sample.
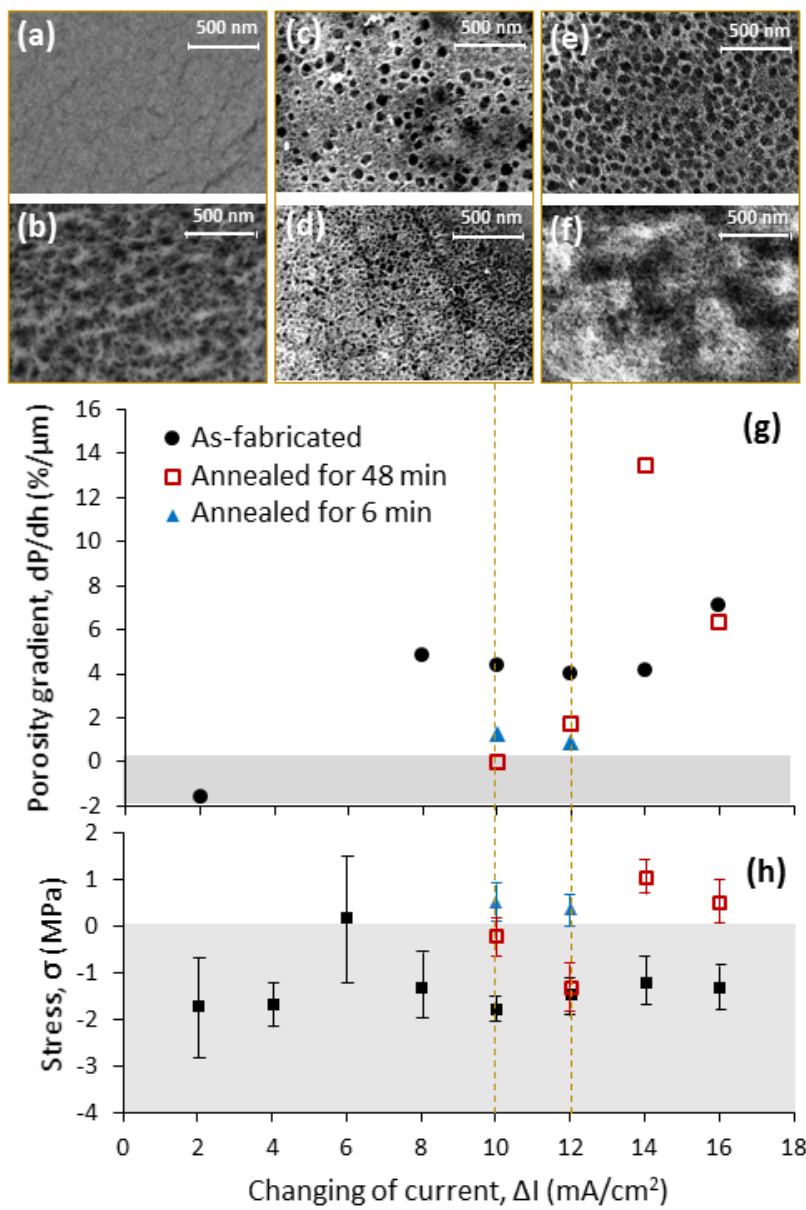

Figure 4: (a) \& (b) SEM images of top side and the back side of detached PS using $\Delta I=0\left(I=20 \mathrm{~mA} / \mathrm{cm}^{2}\right)$, (c) \& (d) SEM images of released PS using $\Delta I=10 \mathrm{~mA} / \mathrm{cm}^{2}$ and (e) \& (f) $\Delta I$ $=12 \mathrm{~mA} / \mathrm{cm}^{2}$. (g) The porosity gradient and $(\mathrm{h})$ the residual stress at different varied current densities.

To test the flatness of PS microbeam using $\Delta \mathrm{I}=10$ $\mathrm{mA} / \mathrm{cm}^{2}$, the surface profile was taken using an optical profilometer. Fig. 5 shows that the variation of the peak to valley (PV) of a microbeam of dimension of $400 \times 20 \mu \mathrm{m}$ is $200 \mathrm{~nm}$ comparing to $340 \mathrm{~nm}$ using a previous approach of an exponential current recipe by Xiao et al. [2]. The yield using our approach is almost $80 \%$. The physical thickness of the PS microstructures is $2.1 \mu \mathrm{m}$ which was measured using Dektak. The air gap created during subsequent masking, electropolishing and critical point drying, was $3.2 \mu \mathrm{m}$, as shown in Fig.5. This result is strong evidence for 
the success of the stress compensation method presented here. Given that the film's Young's modulus is in the range from 1 to $5 \mathrm{GPa}$, even the smallest amount of stress $(|\sigma|<1 \mathrm{MPa})$ can lead film deformation and damage.

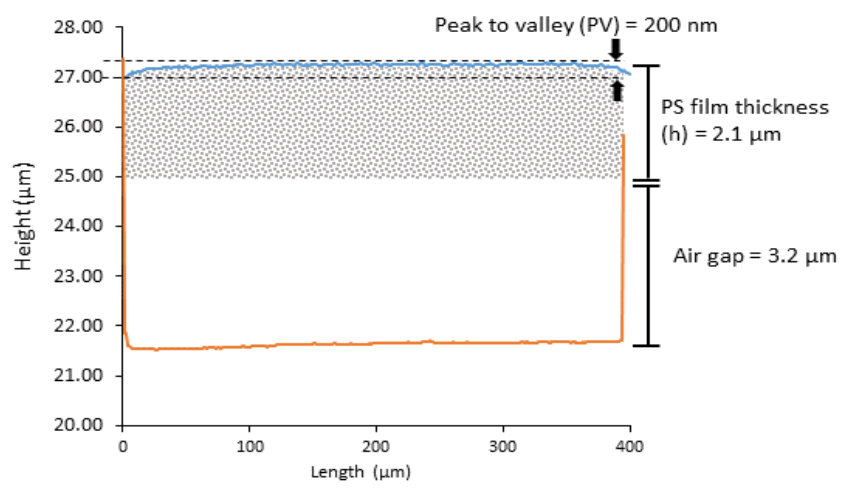

Figure 5: Surface profile of PS microbeam of dimension of $400 \times 20 \mu \mathrm{m}$ using the recipe $\Delta \mathrm{I}=10 \mathrm{~mA} / \mathrm{cm}^{2}$.

\section{Conclusion}

The porosity profile along the depth was investigated by fabricating different PS films using a constant current of I = $10 \mathrm{~mA} / \mathrm{cm}^{2}$ at different thicknesses. The results suggest that the porosity is higher at the Si/PS interface than at the top surface. SEM images demonstrated the size and distribution of pores on the top surface of PS films.

Subsequently, the porosity was compensated by decreasing the current over the anodization duration, starting at $20 \mathrm{~mA} / \mathrm{cm}^{2}$. The porosity gradient along with the residual stress was measured for each recipe. SEM images showed the porosity at both top side and back side of released PS structures. Results showed that decreasing the current from $20 \mathrm{~mA} / \mathrm{cm}^{2}$ and ending at $10 \mathrm{~mA} / \mathrm{cm}^{2}$ produced a good yield and a flatness of about $\mathrm{PV}=200 \mathrm{~nm}$. This work is an important contribution towards the fabrication of released (tunable) resonant cavity optical filters based on PS.

\section{Acknowledgements}

We acknowledge the support from the University of Western Australia (UWA), the Australian Research Council, Lloyd's Register Foundation Postgraduate Scholarship, Western Australian Node of the Australian National Fabrication Facility, and Centre for Microscopy, Characterisation and Analysis and the WA State Government.

\section{References}

[1] M. Nedeljkovic et al., Silicon photonic devices and platforms for the mid-infrared, Opt. Mater. Express, 2013, 3, p 1205. [2] X. Sun, G. Parish and A. Keating, Fabrication of uniform porosity, all-porous-silicon microstructures and stress/stress gradient control J. Micromech. Microeng., 2017, 27, p 044001. [3] K. Valalaki, P. Benech and A. G. Nassiopoulou, High seebeck coefficient of porous silicon: Study of the porosity dependence, Nanoscale Res. Lett., 2016, 11:201.

[4] T. James, A. Keating, G. Parish and C. Musca, Low temperature $\mathrm{N}_{2}$-based passivation technique for porous silicon thin films. Solid State Commun., 2009, 149, p 1322.

[5] D. Bruggeman, Berechnung verschiedener physikalischer Konstanten von heterogenen Substanzen. I.

Dielektrizitätskonstanten und Leitfähigkeiten der Mischkörper aus isotropen Substanzen, Ann. Phys., 1935, 416, p 636.

[6] X. Sun, A. Keating, and G. Parish, Stress control of porous silicon films for microelectromechanical systems, Microporous and Mesoporous Mater., 2015, 218, p 88.

[7] N. Al-Temeeme and G. Muhammed, Study the effect of irradiation time and HF concentration on porosity of porous silicon and study some of the electrical properties of its based device, Adv. Mate. Phys. Chem., 2012, 2(01), p 55.

[8] K. Kulathuraan, K. Mohanraj, B. Natarajan, Structural, optical and electrical characterization of nanostructured porous silicon: Effect of current density, Spectrochim Acta A Mol Biomol Spectrosc., 2016, 5, p 152. 